\title{
Exclusion of Linkage between the Collagenase Gene and Generalized Recessive Dystrophic Epidermolysis Bullosa Phenotype
}

\author{
Alain Hovnanian, ${ }^{\star *}$ Philippe Duquesnoy, ${ }^{\star}$ Serge Amselem, ${ }^{\ddagger}$ Claudine Blanchet-Bardon, $*$ \\ Mark Lathrop," Louis Dubertret, * and Michel Goossens* \\ *Service de Dermatologie, Hopital Saint-Louis, 75475 Paris cedex 10; ${ }^{\ddagger}$ Service de Biochimie and INSERM U. 91, \\ C.H.U. Henri Mondor, 94010 Créteil; and ${ }^{\$}$ Centre d'Etude du Polymorphisme Humain, 75475 Paris cedex 10, France
}

\begin{abstract}
Generalized recessive dystrophic epidermolysis bullosa (RDEB) is a severe inherited autosomal disease characterized by dermolytic blister formation. Enhanced collagenase and/or abnormal collagenase have been reported in skin from affected patients, suggesting that collagenase could be responsible for the absence of anchoring fibrils in this disorder. We used a genetic linkage approach to test the hypothesis that this disease is due to a defect in the collagenase gene in nine affected families. Analysis of amplified genomic DNA fragments of the collagenase gene by means of denaturing gradient gel electrophoresis (DGGE) allowed us to detect intragenic polymorphisms, which were subsequently characterized by direct genomic sequencing. Segregation analysis of these polymorphic sites showed exclusion of linkage between the collagenase gene and generalized RDEB phenotype in a family with consanguineous parents and three affected children. However, the possibility of linkage with the collagenase gene in the other eight families tested could not be excluded. The genetic markers described here provide a tool for investigating genetic linkage in other affected families. Overall, our results show that generalized RDEB can be caused by a defect in a gene other than the collagenase gene, and support the hypothesis that the genetic defect lies in abnormal anchoring fibril formation. (J. Clin. Invest. 1991.88:17161721.) Key words: intragenic polymorphism • polymerase chain reaction • denaturing gradient gel electrophoresis • direct genomic sequencing $\bullet$ lod score
\end{abstract}

\section{Introduction}

Epidermolysis bullosa (EB) ${ }^{1}$ is a group of rare genetic diseases responsible for skin blistering in response to mild trauma. The generalized, dystrophic type of EB (i.e., Hallopeau-Siemens variant, MIM No. 226600) is a severe autosomal recessive con-

Address correspondence to Alain Hovnanian, M.D., Service de Biochimie and INSERM U.91 C.H.U. Henri Mondor, 51, avenue du Maréchal de Lattre de Tassigny, 94010 Créteil, France. 1991.

Received for publication 18 March 1991 and in revised form 11 June

1. Abbreviations used in this paper: $\mathrm{AF}$, anchoring fibrils; $\mathrm{DGGE}$, denaturing gradient gel electrophoresis; EB, Epidermolysis bullosa; PCR, polymerase chain reaction; RDEB, recessive dystrophic epidermolysis bullosa.

J. Clin. Invest.

(c) The American Society for Clinical Investigation, Inc.

$0021-9738 / 91 / 11 / 1716 / 06 \$ 2.00$

Volume 88, November 1991, 1716-1721 dition, characterized by early, extensive skin blistering, with dystrophic healing of the hands and feet, and nail and mucous membrane involvement $(1,2)$. In this kind of $\mathrm{EB}$, the blister occurs between the epidermis and papillary dermis, just beneath the lamina densa. Ultrastructural analyses have shown collagen degeneration, with phagocytosis of collagen fibrils by macrophages in the upper dermis, associated with the absence or rudimentary appearance of anchoring fibrils $(3,4)$. These morphologic findings suggested that recessive dystrophic epidermolysis bullosa (RDEB) might be caused either by excessive collagenase activity resulting in connective tissue destruction and decreased or absent anchoring fibrils, or by a primary defect in anchoring fibril formation (5).

A number of in vivo and in vitro biochemical studies supported the hypothesis that enhanced collagenase activity was of pathologic importance in RDEB. Increased collagenase activity was first reported in explant cultures of blistered skin from RDEB patients $(6,7)$; subsequent in vivo investigations found high tissue levels of collagenase in both blistered and clinically uninvolved skin from patients with RDEB (8). In addition, cultures of fibroblasts from patients with RDEB demonstrated an increased capacity to synthesize and secrete collagenase (911). Because the other inherited forms of EB did not display these abnormalities, it was suggested that collagenase overexpression was a specific biochemical feature of RDEB (12), and that the determination of collagenase activity and concentrations in fetal skin fibroblast culture supernatant could be helpful in prenatal diagnosis (13). Furthermore, phenytoin, an agent that inhibits collagenase synthesis, has been used in the treatment of RDEB patients with inconclusive results $(14,15)$.

The possibility that a structural gene mutation might give rise to a mutant form of collagenase was raised by Bauer (1618) who found that cultured skin fibroblasts from four RDEB patients produced structurally abnormal collagenase with altered biochemical properties. Moreover, the recent demonstration that collagenase is able to degrade type VII collagen (19), the major component of anchoring fibrils (AF) (20), suggested that excessive collagenase activity could account for the absence of AF in this disease.

On the other hand, recent immunofluorescence and immunoelectron microscopy studies have demonstrated abnor$\mathrm{mal}$ in vivo and in vitro expression of type VII collagen in the skin of RDEB patients (21-27). However, whether blister formation in these subjects is related to a primary defect in the gene coding for type VII collagen, or to an excessive degradation of AF by collagenase remains to be determined (28-31).

As a first step in investigating the genetic basis of this disease, we used a genetic linkage approach to test the hypothesis that it results from a defect in the collagenase gene. In this study, we characterized intragenic polymorphisms, and analyzed the segregation of the collagenase haplotypes among the 
Table I. Ethnic Origin, Evidence of Consanguinity, and Number of Affected Offspring in the Nine RDEB Families Studied

\begin{tabular}{|c|c|c|c|c|c|c|c|c|c|}
\hline & \multicolumn{9}{|c|}{ Family } \\
\hline & I & II & III & IV & $\mathbf{v}$ & VI & VII & VIII & IX \\
\hline Origin & Tunisian & Turkish & French & Kurdish & Pakistani & French & Tunisian & French & Portuguese \\
\hline $\begin{array}{l}\text { Consanguinity } \\
\text { No. offspring with EB/ }\end{array}$ & + & - & - & - & + & - & - & - & - \\
\hline $\begin{array}{l}\text { No. offspring with EB/ } \\
\text { No. offspring }\end{array}$ & $3 / 4$ & $1 / 3$ & $1 / 2$ & $1 / 4$ & $1 / 2$ & $1 / 3$ & $1 / 4$ & $2 / 2$ & $2 / 3$ \\
\hline Lod score $(\theta=0.00)$ & $-\infty$ & -0.35 & -0.18 & 0.07 & 0.12 & 0.25 & 0.37 & 0.60 & 0.73 \\
\hline
\end{tabular}

Lod scores between the collagenase gene locus and RDEB were calculated in each family at a recombination fraction $(\theta)$ of 0.00 .

members of nine RDEB families. Our results provide the first genetic evidence excluding linkage between the collagenase gene and the RDEB phenotype in a family with consanguineous parents.

\section{Methods}

\section{Patients}

We studied nine unrelated families with generalized RDEB (Table I). Family I was of Tunisian origin with consanguineous parents and three affected siblings out of four. Consanguinity was also found in family $\mathrm{V}$ of Pakistani extraction who had one affected child out of two. In the other families (II-IV and VI-IX), there was no evidence for consanguinity. Generalized RDEB was diagnosed on the basis of clinical and ultrastructural findings.

\section{Amplification of genomic DNA by the polymerase chain reaction}

Genomic DNA was prepared from peripheral blood leukocytes and amplified as previously described (32) with some modifications. Briefly, genomic DNA $(1 \mu \mathrm{g})$ was subjected to amplification in a $100-\mu \mathrm{l}$ reaction mixture containing $50 \mathrm{mM} \mathrm{KCl}, 10 \mathrm{mM}$ Tris- $\mathrm{HCl}(\mathrm{pH} 8.3)$, $15 \mathrm{mM} \mathrm{MgCl} 2,0.01 \%$ (wt/vol) gelatin, $200 \mathrm{mM}$ of each deoxynucleotide triphosphate, $10 \mathrm{pmol}$ of each primer and $1 \mathrm{U}$ of Taq polymerase (Perkin-Elmer Corp., Pomona, CA). 30 cycles were performed with a DNA thermal cycler (Perkin-Elmer Corp.), each cycle consisting of 1 min of denaturation at $94^{\circ} \mathrm{C}, 30 \mathrm{~s}$ of annealing at $55^{\circ} \mathrm{C}$, and $1 \mathrm{~min}$ of extension at $72^{\circ} \mathrm{C}$. To enhance heteroduplex formation, the PCR cycles were followed by a 10 -min denaturation step at $94^{\circ} \mathrm{C}$ and a $1-\mathrm{h}$ annealing step at $56^{\circ} \mathrm{C}$. A 1,605 -bp segment of the collagenase gene spanning exons 4-6 was amplified using two 20-base oligonucleotide primers ( $\mathrm{p} 1$ and $\mathrm{p} 2$ ) complementary to the published sequence of the collagenase cDNA (33). Subsequent amplifications of internal regions within this fragment were performed using different sets of primers (p1-p8, p1-p6, p1-p4, and p5-p6). Their positions and nucleotide sequences are shown in Fig. $1 B$ and Table II.

\section{Analysis of the PCR products}

Gel electrophoresis. The amplified products were analyzed by means of electrophoresis on $6 \%$ polyacrylamide gels and denaturing gradient gels according to a previously described method (34) which permits the detection of single basepair variations within a double-stranded DNA fragment run on a gel containing an increasing denaturant concentration (35). The melting of the lowest temperature melting domain of the fragment retards its mobility, thus permitting the separation of DNA fragments whose nucleotide sequences differ within their first melting domain. The electrophoretic pattern observed after denaturing gradient gel electrophoresis (DGGE) is formed of bands that correspond to homoduplexes (resulting in reassociation of complementary strands of one allele), and heteroduplexes formed between two different alleles. One-fifth of each PCR product was electrophoresed on a vertical $6.5 \%$ polyacrylamide gel with a linearly increasing $10-60 \%$ denaturing gradient $(100 \%$ denaturant $=7 \mathrm{M}$ urea and $40 \%$ formamide) parallel to the direction of the electrophoresis. The gel was run at $160 \mathrm{~V}$ for $5 \mathrm{~h}$, stained with ethidium bromide, and photographed.
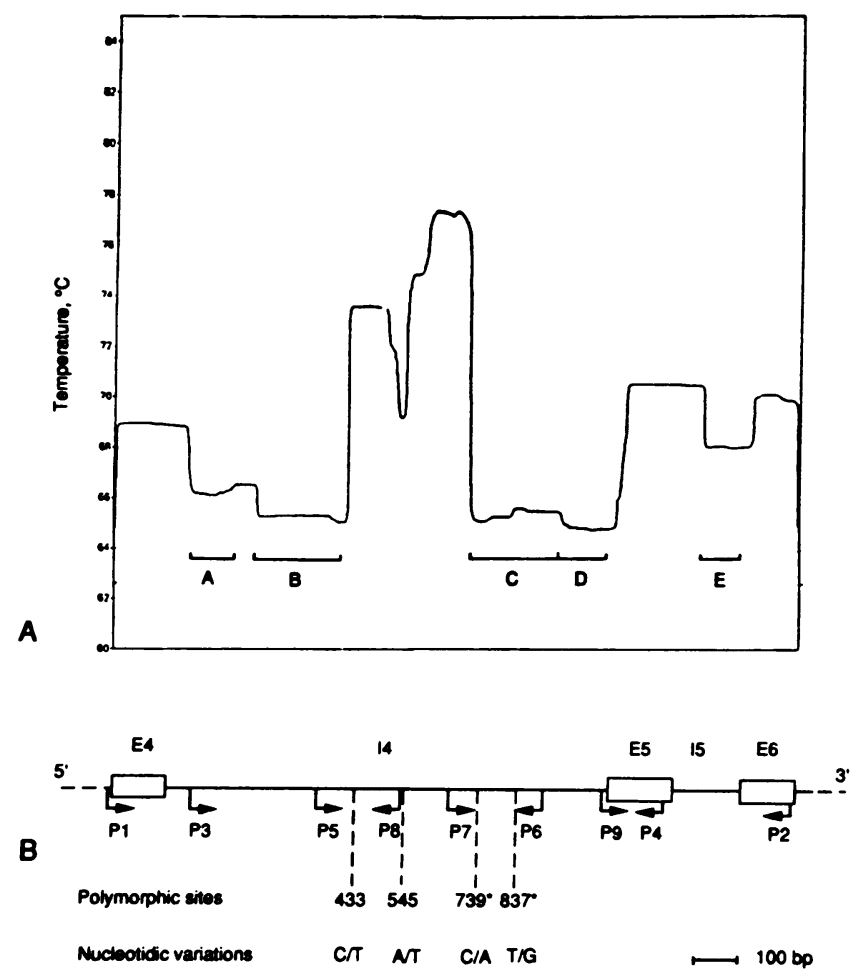

Figure 1. Melting map of the PCR-amplified fragment of the collagenase gene spanning exons 4-6 $(A)$, and the corresponding schematic representation $(B) .(A)$ The curve represents the midmelting temperature $\left(T_{\mathrm{m}}\right.$, temperature at which the probability of melting of a basepair is 50\%) plotted as a function of the base pair position in the DNA sequence by using Lerman's Melt 87 program. The lowest melting domains (A-E) are indicated. The $x$-axis corresponds to the nucleotide sequence from 228 within intron 3 , to 109 within exon 6 . The $y$-axis represents the midmelting temperature. $(B)$ Diagram of this segment of the collagenase gene. The coding sequences (E4-E6) are represented by open boxes and the intronic sequences (I4 and I5) by the horizontal line. The arrows indicate the position and orientation of the oligonucleotide primers used in DNA amplification and sequencing reactions. The position and the nature of the nucleotide polymorphisms are indicated. The numbers refer to their nucleotide position within the fourth intron. Asterisk denotes two polymorphic sites explored in the denaturing conditions we used. 
Table II. DNA Sequences and Position of the Oligonucleotide Primers Used in Polymerase Chain Reaction Assays and Sequencing Reactions

\begin{tabular}{|c|c|c|c|c|c|}
\hline \multirow[t]{2}{*}{ P1 } & \multirow[t]{2}{*}{$5^{\prime}$} & CAGATCATCGGGACAACTCT $3^{\prime}$ & \multirow[t]{2}{*}{ P6 } & \multirow[t]{2}{*}{$5^{\prime}$} & TTTCCGAAGCAGAAGGCATG 3' \\
\hline & & I3-228 & & & I4-913 \\
\hline \multirow[t]{2}{*}{$\mathbf{P} 2$} & \multirow[t]{2}{*}{$5^{\prime}$} & AAGAACATCACTTCTCCCCG 3' & \multirow[t]{2}{*}{ P7 } & \multirow[t]{2}{*}{$5^{\prime}$} & GCAAGCTCCGTCTCAAGAAA $3^{\prime}$ \\
\hline & & E6-109 & & & I4-681 \\
\hline \multirow[t]{2}{*}{ P3 } & \multirow[t]{2}{*}{$5^{\prime}$} & ATTCATAGAAGTATATATTT $3^{\prime}$ & \multirow[t]{2}{*}{ P8 } & \multirow[t]{2}{*}{$5^{\prime}$} & CGTGTGCCACCATGCCTGTC 3' \\
\hline & & I4-86 I4-105 & & & I4-550 \\
\hline \multirow[t]{2}{*}{ P4 } & \multirow[t]{2}{*}{$5^{\prime}$} & CTGAACATCACCACTGAAGG $3^{\prime}$ & \multirow[t]{2}{*}{ P9 } & \multirow[t]{2}{*}{$5^{\prime}$} & GCCTTTTTAGAGTACAACTT 3' \\
\hline & & E5-116 & & & I4-1044 \\
\hline \multirow[t]{2}{*}{ P5 } & \multirow[t]{2}{*}{$5^{\prime}$} & \multicolumn{4}{|l|}{ TCCAGCAGTCAACATTTATC $3^{\prime}$} \\
\hline & & I4-364 & & & \\
\hline
\end{tabular}

Numbers refer to the nucleotide position within intronic (I) and exonic (E) regions.

Direct sequencing of amplified DNA. Single-strand DNA suitable for sequencing was generated in the polymerase chain reaction using unequal amounts of primers $\mathrm{p} 1$ and $\mathrm{p} 2(50 \mathrm{pmol} / 1 \mathrm{pmol})$ as previously described (36). The product of asymmetric amplification was concentrated using a Centricon 100 (Amicon Corp., Danvers, MA) and an aliquot of this material was used as DNA template for sequencing by means of the dideoxynucleotide termination method (37) using a Sequenase kit (United States Biochemical Corp., Cleveland, $\mathrm{OH}$ ) and $\alpha_{-}{ }^{35} \mathrm{~S}$ dATP $(400 \mathrm{Ci} / \mathrm{mmol})$ (Amersham Corp., Arlington Heights, IL). The oligonucleotides used for sequencing (primers p1-p9) (Fig. $1 B$ ) were synthesized on a 380B DNA synthesizer (Applied Biosystems, Inc., Foster City, CA).

\section{Computer analysis}

Computational simulation of DNA melting of the plp2-amplified gene segment was performed using Lerman's Melt 87 program which predicts the melting properties of a DNA fragment according to its nucleotide sequence (38). The sets of primers used to test the different fusion domains were selected according to their position and orientation on the melting map (Fig. 1). The denaturing conditions resulting in optimal resolution and heteroduplex formation were determined by displacement calculation using Lerman's SQHTX program (38).

\section{Linkage analysis}

Linkage analysis was performed using the LINKAGE program (39). Lod score ${ }^{2}$ calculations were performed by designating RDEB as a recessive disease with complete penetrance, taking into account the consanguinity in families I and V. Heterogeneity analysis was performed with the Homog program (40).

\section{Results}

Because no collagenase gene restriction fragment length polymorphism has been described, we looked for nucleotide polymorphisms useful in linkage studies by means of DGGE. We selected two primers that allowed the amplification of a 1,605bp region spanning exons 4-6, thus including exon 5, which encodes the catalytic site of the enzyme (41), and its two flanking introns.

Detection of polymorphic sites within the collagenase gene An amplified product of expected size was obtained upon PCR amplification of the 1,605-bp DNA fragment in all subjects

2. A lod-score is defined as the $\log 10$ of the odds that the disease and marker loci are linked rather than unlinked at a specific recombination fraction. from family I, ruling out a deletion within this fragment (data not shown). When these amplified products were tested for DNA melting polymorphisms by means of DGGE, different patterns were observed among the members of this family, suggesting the existence of several nucleotide sequence polymorphisms. To correlate the melting patterns observed with the nucleotide sequence of the fragment, we determined the complete sequence of this DNA fragment in one member of family I by direct genomic sequencing. The melting behavior of the fragment (Fig. $1 A$ ) was predicted using Lerman's computer program. The fusion map contains five low melting domains (A-E) which were analyzed using different sets of primers (p1p8, p1-p6, p1-p4, and p5-p6) (Fig. $1 B$ ) to identify the domain that contains the polymorphism. As the PCR products generated with primers p5-p6 (550-bp fragment) (Figs. $1 B$ and $2 B$ ) and p1-p2 (full-length 1,605-bp fragment) displayed similar DGGE patterns, we restricted our analysis to this limited segment of the collagenase gene in all the RDEB patients and their families. The pattern obtained with primers p5-p6 in family $I$ is shown in Fig. 2 C. Direct genomic sequencing of this 550-bp amplified product allowed us to characterize four polymorphic sites within the fourth intron. Two of them, located in the early melting region of the fragment (domain C) (Fig. 1), were likely to be responsible for the DGGE pattern observed. These C-to$A$ and T-to-G substitutions (at positions 739 and 837, respectively) (Fig. 3) did not affect any known restriction enzyme recognition sites. The two other polymorphic sites (C-to-T substitution at position 433, and A-to-T substitution at position 545 ) could not be detected by DGGE because they were located in a highly stable region of the fourth intron (Fig. 1).

\section{Collagenase gene haplotypes and their use in linkage analysis}

These four polymorphic sites defined seven haplotypes that are summarized in Table III; their distribution in the RDEB families is shown in Fig. 4. The parental phases of the collagenase alleles were established by segregation analysis within each family. The linkage between the disease and the collagenase alleles in each family was tested by lod score analysis, and the results are given for a recombination fraction $(\theta)$ of 0.00 (Table I).

Family I: exclusion of linkage. Family I (consanguineous parents and three affected siblings) was found to be informative for the polymorphic sites tested (i.e., the parents were heterozygous at the collagenase locus, with one differing haplotype) (Fig. $2 C$ ). In this family, several features allowed us to exclude 
A

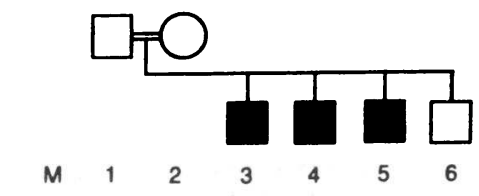

B
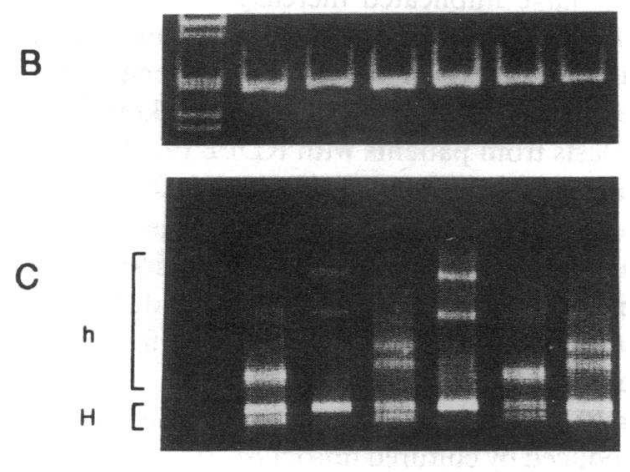

D

bc bd cd bd bc cd

Figure 2. Partial pedigree of family I $(A)$; nondenaturing gel electrophoresis of PCR-amplified genomic fragment of the collagenase gene obtained with primers p5-p6 $(B)$, corresponding DGGE $(C)$, and collagenase haplotypes established by direct sequencing $(D)$. $(A)$ Squares and circles represent males and females, respectively. Solid symbols represent subjects with clinical disease, the open symbols a normal or heterozygous individual, and the double bar between parents denotes a consanguineous union. $(B)$ Electrophoresis in a nondenaturing $6 \%$ acrylamide gel of DNA samples amplified with p5-p6 primers revealed a single 550 -bp band in all members of the family. $(M)$ molecular weight markers given by the 1-kb ladder (top to bottom, 1600 , $1000,517-506,396,344 \mathrm{bp}$ ). (C) DGGE patterns observed with this p5-p6 amplified fragment of the collagenase gene in the family members showing homoduplexes $(H)$ and heteroduplexes $(h)$ with different stability. The family was informative for these DNA melting polymorphisms (i.e., the parents were heterozygous and had different heteroduplexes). The three affected siblings $(3,4$, and 5$)$ were heterozygous at the collagenase locus, inherited different collagenase alleles (cd, bd, and bc, respectively), and the first affected sibling displayed the same genotype as his clinically unaffected brother $(6, \mathrm{~cd})$. (D) Sequence analysis of this gene fragment showed that each of the three collagenase haplotypes $(b-d)$ could cosegregate with the RDEB phenotype.

genetic linkage between the collagenase gene and the RDEB phenotype. First, the three affected siblings were heterozygous at the collagenase locus. This is not consistent with the notion that in rare recessive inherited diseases, affected children from consanguineous families are expected to be homozygous for the defective allele inherited from a common ancestor (42). Second, if there were linkage between the collagenase gene and RDEB, all the affected children within a given family should have the same genotype at the collagenase locus. However, the pedigree of family I shows that the three affected siblings had different collagenase genotypes (3-cd; 4-bd; 5-bc) (Fig. 2 D). Furthermore, the first affected sibling (3-cd) had the same collagenase genotype as his phenotypically normal brother (6-cd). Because the DNA polymorphisms used in this study were intragenic, the risk of recombination between these polymorphic sites and the collagenase gene was very unlikely. Consequently, we excluded genetic linkage between the collagenase gene and the RDEB phenotype in this family.

Families II-IX. No obligate recombination events were detected in families II-IX. Lod scores calculated to test the hypothesis of linkage to the collagenase gene in individual fami-
A

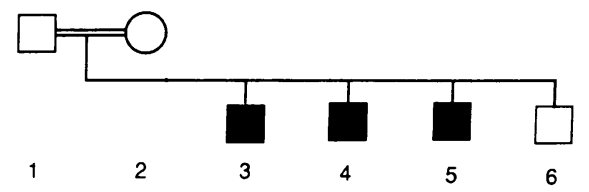

B

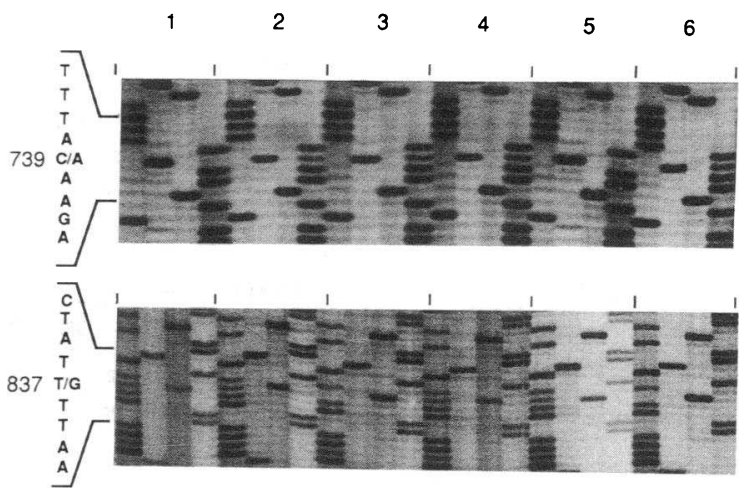

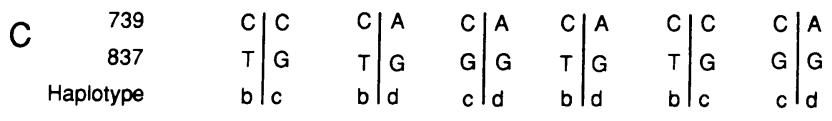

Figure 3. Partial pedigree of family I $(A)$, characterization of polymorphic sites by direct sequence analysis $(B)$, and corresponding collagenase haplotypes $(C)$. The sequences shown are those of the positive strand obtained by using primers $\mathrm{p} 5$ and $\mathrm{p} 7$ in the sequencing reaction. The order of nucleotides is TCGA. The C-to-A and T-to-G substitutions at positions 739 and 837, respectively, define two polymorphic sites within the fourth intron. The corresponding collagenase haplotypes in this family $(b-d)$ are associated with both the RDEB phenotype and the normal phenotype.

lies ranged from -0.35 to 0.73 at $\theta=0.00$ (Table I). Overall, the maximum lod score when family I was included was 0.78 at $\theta$. $=0.10(P<0.05)$. The test of heterogeneity (i.e., a test of linkage within a subset of families) was not significant $(P=0.25)$.

\section{Discussion}

In this study, we tested the hypothesis that generalized RDEB is due to a defect in the collagenase gene. Nine families with at least one child suffering from generalized RDEB were studied. We focused on the molecular analysis of a 1,605-bp fragment of the collagenase gene which contained two interesting se-

Table III. Collagenase Haplotypes in the Nine RDEB Families Studied

\begin{tabular}{|c|c|c|c|c|c|c|}
\hline \multirow{3}{*}{ Haplotype } & \multicolumn{4}{|c|}{ Location of polymorphic sites } & \multirow{2}{*}{\multicolumn{2}{|c|}{$\begin{array}{l}\text { Chromo- } \\
\text { somes } \\
\text { studied } \\
(n=90)\end{array}$}} \\
\hline & \multirow[t]{2}{*}{ IVSnt433 } & \multirow[t]{2}{*}{ IVSnt545 } & \multirow[t]{2}{*}{ IVSnt739 } & \multirow[t]{2}{*}{ IVSnt837 } & & \\
\hline & & & & & No. & $\%$ \\
\hline $\mathbf{a}$ & $\mathrm{C}$ & A & $\mathrm{C}$ & G & 25 & 27.7 \\
\hline b & $\mathrm{C}$ & $\mathbf{A}$ & C & $\mathrm{T}$ & 24 & 26.0 \\
\hline c & $\mathrm{C}$ & $\mathbf{T}$ & $\mathrm{C}$ & $\mathbf{G}$ & 15 & 16.6 \\
\hline d & $\mathrm{T}$ & $\mathbf{A}$ & $\mathbf{A}$ & $\mathbf{G}$ & 15 & 16.6 \\
\hline e & $\mathrm{C}$ & $\mathbf{T}$ & C & $\mathbf{T}$ & 6 & 6.6 \\
\hline f & $\mathrm{C}$ & $\mathbf{T}$ & $\mathbf{A}$ & G & 3 & 3.3 \\
\hline $\mathbf{g}$ & $\mathbf{T}$ & A & A & $\mathrm{T}$ & 2 & 2.2 \\
\hline
\end{tabular}

Location of the polymorphic sites refers to the nucleotide (nt) position within the fourth intervening sequence (IVS) of the collagenase gene. 

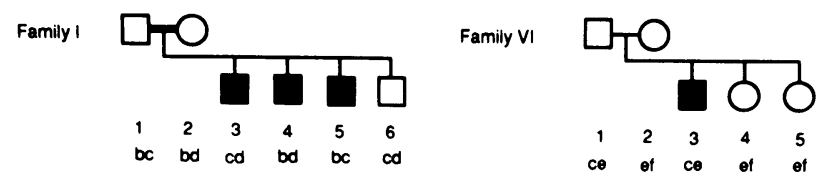

Family 11

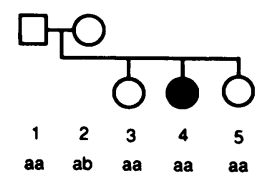

Family III

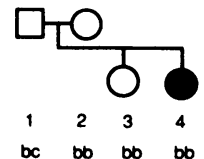

Family IV

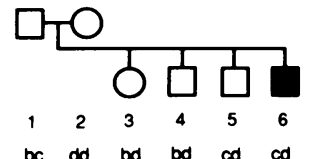

Family IX


by be bo bo by

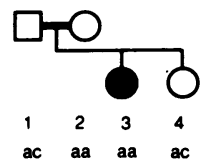

Figure 4. Haplotypes of the collagenase gene in RDEB patients and their families. The numbers indicate the individual in the family, and the letters the haplotypes for the collagenase gene. The designation of the haplotypes is shown in Table III.

quences, i.e., the sequence (exon 5) coding for the catalytic domain, thus a possible target for deleterious mutations, and its adjacent intronic regions (introns 4 and 6 ) which could display nucleotide polymorphisms.

DGGE analysis of this amplified gene fragment in one family with consanguineous parents and three affected children revealed multiallele polymorphisms that we subsequently characterized by direct genomic sequencing. These polymorphisms did not cosegregate with the RDEB phenotype, excluding linkage of this gene to the disease in family I. Though statistically very unlikely, the possibility of a dominant form of the disease in this kindred, resulting from a germ line mutation in three different gametes, could have been considered. However, this possibility was formally excluded given the severe mittenlike deformities of the hands and feet observed in the oldest and most severely affected offspring: this clinical feature has not been described in any dominant form of dystrophic epidermolysis bullosa (EB), but is characteristic of the generalized recessive form of dystrophic EB (Hallopeau-Siemens variant).

Using the same genetic approach, we performed linkage analysis between the RDEB phenotype and the collagenase haplotypes in eight other affected families. The role of a defect in the collagenase gene in the disease could not be ruled out in these families in which no obligate recombination events were found. Indeed, the maximum lod score was 0.78 at $\theta=0.10$ for all the families (including family I). It should be recalled that the usual requirement for significant linkage (a lod score $>3$ ) applies to linkage between a random marker and the disease locus, and that a less stringent criterion can be applied to the

examination of a candidate gene, in this case, collagenase. The result of the formal test for heterogeneity, in which the hypothesis of linkage within a subgroup of families is examined, was not statistically significant. These results suggest that the study of linkage between the collagenase gene and the RDEB phenotype in further affected families would be warranted.

Several authors have implicated increased (6-12, 29-31) and/or abnormal collagenase $(12,16-18)$ in the pathogenesis of blister formation in RDEB. In particular, collagenase overexpression has been found in clinically uninvolved skin (8) and in cultured fibroblasts from patients with RDEB (9). This biochemical trait was considered to be specific for this form of EB, and was shown to persist through several cell passages, suggesting that it was genetically determined (9). In addition, the recent demonstration that collagenase is able to degrade type VII collagen (19), the major component of anchoring fibrils (20), reinforced the candidate gene status of collagenase.

Other authors reported the isolation of a structural variant of collagenase produced by cultured fibroblasts from four unrelated patients with generalized RDEB (16-18). This abnormal form of the enzyme was expressed at high levels and exhibited increased thermolability, decreased calcium affinity, and a slightly different global amino acid composition, but normal catalytic activity towards types I and III collagen (12, 16-18). The catalytic activity of the mutant form towards type VII collagen has not been studied, and its cDNA sequence has not been published. Overall, these data suggested that RDEB might be caused by a defect in the regulation of the collagenase gene, or in the structure of the gene itself.

The candidate gene approach we used excluded any linkage between the collagenase gene and generalized RDEB in one of the affected families studied. Indeed, our strategy, which is based on linkage analysis, allowed us to test the involvement of the genetic region encompassing the collagenase gene in the RDEB phenotype. Though we focused our analysis on a region of the gene spanning the active site of the collagenase, our results are not contingent upon the location of a putative defect within the collagenase gene.

So far, we cannot exclude linkage between the collagenase gene and the disease in all other affected families because RDEB is likely to be a heterogeneous disorder $(2,43,44)$. Indeed, Bauer reported that collagenase production by cultured skin fibroblasts was only enhanced in eight of 10 patients with RDEB (9), and in a recent study of Scandinavian RDEB patients, collagenase overexpression was only found in cultured fibroblasts from four of 18 RDEB patients (44). Similarly, we found that cultured fibroblasts from only two of the three affected siblings in family I expressed high levels of collagenase mRNA (data not shown), suggesting that collagenase mRNA overexpression was not the primary defect in this family.

Furthermore, our results demonstrate that in RDEB family I, the absence of anchoring fibrils was not due to a primary defect in the collagenase gene. An alternative explanation for the pathophysiology is an abnormality in anchoring fibril formation. The different steps in the biosynthesis of anchoring fibrils are poorly understood, but the description of type VII collagen as the major component of anchoring fibrils provides a new element in the search for the basic defect in RDEB.

This study demonstrates that generalized RDEB phenotype can be caused by defects in a gene other than the collagenase gene. Because genetic heterogeneity cannot be excluded, the intragenic polymorphic sites within the collagenase gene that we have characterized provide useful DNA markers to test for 
the involvement of this gene in other RDEB families, particularly those with abnormal collagenase expression or structure. The type VII collagen gene (or another gene encoding a protein essential for anchoring fibril formation), should be the next candidate gene to investigate.

\section{Acknowledgments}

We are grateful to Dr. B. Bertaux, Prof. C. Beylot, Dr. R. Breathnach, Dr. M. Brohmi, Mrs. F. Froissart, Dr. G. Gatin, Prof. E. Grosshans, Dr. R. Grossman, Dr. A. Hervé, Dr. C. Julier, Prof. L. S. Lerman, Dr. M. Mitjavila, Prof. Y. de Prost, Dr. C. Prost, Prof. J. Revuz, and Prof. M. Vidaud for their helpful collaboration.

This work was supported by the Institut National de la Santé et de la Recherche Médicale (INSERM) and the Centre National de la Recherche Scientifique (CNRS).

\section{References}

1. Cooper, T. W., and E. A. Bauer. 1984. Epidermolysis bullosa: a review. Pediatr. Dermatol. 1:181-188.

2. Fine, J. D., E. A. Bauer, R. A. Briggaman, D. M. Carter, R. A. Eady, N. B. Esterly, K. A. Holbrook, S. Hurwitz, L. Johnson, A. Lin, R. Pearson, and V. P. Sybert. 1991. Revised clinical and laboratory criteria for subtypes of inherited epidermolysis bullosa. A consensus report by the subcommittee on diagnosis and classification of the national epidermolysis bullosa registry. J. Am. Acad. Dermatol. 24:119-135.

3. Pearson, R. W. 1962. Studies on the pathogenesis of epidermolysis bullosa J. Invest. Dermatol. 39:551-575.

4. Hashimoto, I., U. W. Schnyder, I. Anton-Lamprecht, and T. Gedde-Dahl. 1976. Ultrastructural studies in epidermolysis bullosa hereditaria. III. Recessive dystrophic types with dermolytic blistering (Hallopeau-Siemens types and inverse type). Arch. Dermatol. Res. 256:137-150.

5. Briggaman, R. A., and C. E. Wheeler. 1975. Epidermolysis bullosa dystrophica recessive: a possible role of anchoring fibrils in the pathogenesis. J. Invest. Dermatol. 65:203-211.

6. Eisen, A. Z. 1969. Human skin collagenase: relationship to the pathogenesis of epidermolysis bullosa dystrophica. J. Invest. Dermatol. 52:499-453.

7. Lazarus, G. S. 1972. Collagenase and connective tissue metabolism in epidermolysis bullosa. J. Invest. Dermatol. 58:242-248.

8. Bauer, E. A., T. Gedde-Dahl, and A. Z. Eisen. 1977. The role of human skin collagenase in epidermolysis bullosa. J. Invest. Dermatol. 68:119-124.

9. Bauer, E. A., and A. Z. Eisen. 1978. Recessive dystrophic epidermolysis bullosa: evidence for increased collagenase as a genetic characteristic in cell culture. J. Exp. Med. 148:1378-1387.

10. Valle, K. J., and E. A. Bauer. 1980. Enhanced biosynthesis of human skin collagenase in fibroblast cultures from recessive dystrophic epidermolysis bullosa. J. Clin. Invest. 66:176-187.

11. Kronberger, A., K. J. Valle, A. Z. Eisen, and E. A. Bauer. 1982. Enhanced cell-free translation of human skin collagenase in recessive dystrophic epidermolysis bullosa. J. Invest. Dermatol. 79:208-211.

12. Bauer, E. A. 1986. Collagenase in recessive dystrophic epidermolysis bullosa. Ann. NY Acad. Sci. 460:311-320.

13. Bauer, E. A., M. D. Ludman, J. D. Goldberg, R. L. Berkowitz, and K. A. Holbrook. 1986. Antenatal diagnosis of recessive dystrophic epidermolysis bullosa: collagenase expression in cultured fibroblasts as a biochemical marker. $J$. Invest. Dermatol. 87:597-601.

14. Bauer, E. A., T. W. Cooper, D. R. Tucker, and N. B. Esterly. 1980. Phenytoin therapy of recessive dystrophic epidermolysis bullosa. Clinical trial and proposed mechanism of action on collagenase. N. Engl. J. Med. 303:776781 .

15. Kemmett, D., and G. C. Priestley. 1990. Phenytoin in recessive dystrophic epidermolysis bullosa. J. Dermatol. Treatment. 1:147-149.

16. Bauer, E. A. 1977. Recessive dystrophic epidermolysis bullosa: evidence for an altered collagenase in fibroblast cultures. Proc. Natl. Acad. Sci. USA 74:4646-4650.

17. Stricklin, P. G., H. G. Welgus, and E. A. Bauer. 1982. Human skin collagenase in recessive dystrophic epidermolysis bullosa. Purification of a mutant enzyme from fibroblast cultures. J. Clin. Invest. 69:1373-1383.

18. Bauer, E. A. 1982. Abnormalities in collagenase expression as in vitro markers for recessive dystrophic epidermolysis bullosa. J. Invest. Dermatol. 79:105s-108s.

19. Seltzer, J. L., A. Z. Eisen, E. A. Bauer, N. P. Morris, R. W. Glanville, and R. E. Burgeson. 1989. Cleavage of type VII collagen by interstitial collagenase and type IV collagenase (gelatinase) derived from human skin. J. Biol. Chem. 264:3822-3826.

20. Sakai, L. Y., D. R. Keene, N. P. Morris, and R. E. Burgeson. 1986. Type VII collagen is a major structural component of anchoring fibrils. J. Cell Biol. 103:1577-1586.

21. Heagerty, A. H. M., A. R. Kennedy, I. M. Leigh, P. Purkis, and R. A. J. Eady. 1986. Identification of an epidermal basement membrane defect in recessive forms of dystrophic epidermolysis bullosa by LH7:2 monoclonal antibody: use in diagnosis. Br. J. Dermatol. 115:125-131.

22. Leigh, I. M., R. A. J. Eady, A. H. M. Heagerty, P. E. Purkis, P. A. Whitehead, and R. E. Burgeson. 1988. Type VII collagen is a normal component of epidermal basement membrane, which shows altered expression in recessive dystrophic epidermolysis bullosa. J. Invest. Dermatol. 90:639-642.

23. Bruckner-Tuderman, L., S. Rüegger, B. Odermatt, Y. Mitsuhashi, and U. W. Schnyder. 1988. Lack of type VII collagen in unaffected skin of patients with severe recessive dystrophic epidermolysis bullosa. Dermatologica. 176:5764.

24. Bruckner-Tuderman, L., Y. Mitsuhashi, U. W. Schnyder, and P. Bruckner. 1989. Anchoring fibrils and type VII collagen are absent from skin in severe recessive dystrophic epidermolysis bullosa. J. Invest. Dermatol. 93:3-9.

25. Bruckner-Tuderman, L., K. M. Niemi, M. Kero, U. W. Schnyder, and T.

Reunala. 1990. Type VII collagen is expressed but anchoring fibrils are defective in dystrophic epidermolysis bullosa inversa. Br. J. Dermatol. 122:383-390.

26. Rusenko, K. W., W. R. Gammon, J. D. Fine, and R. A. Briggamann. 1989. The carboxy-terminal domain of type VII collagen is present at the basement membrane in recessive dystrophic epidermolysis bullosa. J. Invest. Dermatol. 92:623-627.

27. Smith, L. T., and V. P. Sybert. 1990. Intra-epidermal retention of type VII collagen in a patient with recessive dystrophic epidermolysis bullosa. J. Invest. Dermatol. 94:261-264.

28. Briggaman, R. A. 1985. Is there any specificity to defects of anchoring fibrils in epidermolysis bullosa dystrophica, and what does this mean in terms of pathogenesis? J. Invest. Dermatol. 84:371-373.

29. Bauer, E. A., and M. Tabas. 1988. A perspective on the role of collagenase in recessive dystrophic epidermolysis bullosa. Arch. Dermatol. 124:734-736.

30. Fine, J. D. 1988. Changing clinical and laboratory concepts in inherited epidermolysis bullosa. Arch. Dermatol. 124:523-526.

31. Bauer, E. A. 1990. The role of proteinases in epidermolysis bullosa: general principles of basement membrane zone remodelling in relationship to the pathogenesis of epidermolysis bullosa. In Epidermolysis Bullosa: A Comprehensive Review of Classification, Management and Laboratory Studies. G. C. Priestley, M. J. Tidman, J. B. Weiss, and R. A. J. Eady, editors. DEBRA/Crowthorne, Berkshire, UK. 141-151.

32. Saiki, R. K., D. H. Gelfand, S. Stoffel, S. J. Scharf, R. Higuchi, G. T. Horn, K. B. Mullis, and H. A. Erlich. 1988. Primer-directed enzymatic amplification of DNA with a thermostable DNA polymerase. Science (Wash. DC). 239:487-491.

33. Goldberg, G. I., S. M. Wilhelm, A. Kronberger, E. A. Bauer, G. A. Grant, and A. Z. Eisen. 1986. Human fibroblast collagenase: complete primary structure and homology to an oncogene transformation-induced rat protein. J. Biol. Chem. 261:6600-6605.

34. Attree, O., D. Vidaud, M. Vidaud, S. Amselem, J. M. Lavergne, and M. Goossens. 1989. Mutations in the catalytic domain of human coagulation factor IX: rapid characterization by direct genomic sequencing of DNA fragments displaying an altered melting behaviour. Genomics. 4:266-272.

35. Myers, R. M., T. Maniatis, and L. S. Lerman. 1987. Detection and localization of single base changes by denaturing gradient gel electrophoresis. Methods Enzymol. 155:501-527.

36. Gyllensten, U. B., and H. A. Erlich. 1988. Generation of single-strand DNA by the polymerase chain reaction and its application to direct sequencing of the HLA-DQA locus. Proc. Natl. Acad. Sci. USA. 85:7652-7656.

37. Sanger, F., S. Nicklen, and A. R. Coulson. 1977. DNA sequencing with chain-terminating inhibitors. Proc. Natl. Acad. Sci. USA. 74:5463-5467.

38. Lerman, L. S., and K. Silverstein. 1987. Computational simulation of DNA melting and its application to denaturing gradient gel electrophoresis. Methods Enzymol. 155:482-501.

39. Lathrop, M. G., and J. M. Lalouel. 1984. Easy calculations of lod scores and genetic risks on small computers. Am. J. Hum. Genet. 36:460-465.

40. Ott, J. 1988. Analysis of Human Genetic Linkage. The Johns Hopkins University Press, Baltimore, MD. 233 pp.

41. Collier, I. E., J. Smith, A. Kronberger, E. A. Bauer, S. M. Wilhelm, A. Z. Eisen, and G. I. Goldberg. 1988. The structure of the human skin fibroblast collagenase gene. J. Biol. Chem. 263:10711-10713.

42. Lander, E. S., and D. Botstein. 1987. Homozygosity mapping: a way to map human recessive traits with the DNA of inbred children. Science (Wash. DC). 236:1567-1570.

43. Gedde-Dahl, T. 1986. Clinical heterogeneity in epidermolysis bullosa: speculations on causation and consequence for research. J. Invest. Dermatol. 86:91-92.

44. Winberg J.-O, T. Gedde-Dahl, and E. A. Bauer. 1989. Collagenase ex pression in skin fibroblasts from families with recessive dystrophic epidermolysis bullosa. J. Invest. Dermatol. 92:82-85. 